\title{
Genetic Variants in the Activation of the Brown-Like Adipocyte Pathway and the Risk for Severe Obesity
}

\author{
Ana Carolina Proença da Fonseca ${ }^{a} \quad$ Guilherme Proença da Fonsecab \\ Bruna Marchesini $^{a} \quad$ Danielle Dutra Voigt ${ }^{b}$ Mario Campos Junior ${ }^{a}$ \\ Verônica Marques Zembrzuski ${ }^{a}$ João Regis Ivar Carneiro ${ }^{c}$ \\ José Firmino Nogueira Neto ${ }^{d}$ Pedro Hernan Cabello ${ }^{a, b}$ \\ Giselda Maria Kalil Cabello ${ }^{a}$ \\ ${ }^{a}$ Human Genetics Laboratory, Oswaldo Cruz Institute/Fiocruz, Rio de Janeiro, Brazil; \\ bHuman Genetics Laboratory, Grande Rio University, Rio de Janeiro, Brazil; \\ ${ }^{c}$ Clementino Fraga Filho University Hospital, Federal University of Rio de Janeiro, \\ Rio de Janeiro, Brazil; ${ }^{d}$ Department of Pathology and Laboratory, Rio de Janeiro State \\ University, Rio de Janeiro, Brazil
}

\section{Keywords}

Polymorphism · Irisin · Severe obesity · UCP1 · PPARGC1A

\begin{abstract}
Background: Regular physical activity has an important role in energy expenditure and combats the development of obesity. During exercise, PPARGC1A is overexpressed, stimulating an increase of the expression of FNDC5. This protein is cleaved to release the hormone irisin, which activates a browning process in white adipose tissue through an increase in UCP1 expression. As a result, irisin leads to mitochondrial heat production and energy expenditure. Objectives: The aim of this study was to investigate whether genetic variants in genes related to browning are associated with severe obesity and obesity-related features. This case-control study comprised 210 individuals with severe obesity (median body mass index [BMI] 45.6 [range 40.5-52.2]) and 191 normal-weight subjects (BMI 22.8 [21.1-23.9]). Methods: Genomic DNA was extracted from peripheral blood and the genotypes of the PPARGC1A (rs8192678, rs3736265, rs2970847, and rs3755863) and UCP1 (rs6536991 and rs12502572) genes were obtained using $\operatorname{Taqman}^{\circledR}$ assay. For the FNDC5 gene, screening of exons $3-5$ as well as their intron-exon boundaries was performed using automatic sequencing. Results: Our results demonstrated that PPARGC1A rs2970847 and UCP1 rs12502572 are associated with severe obesity. Furthermore, these polymorphisms influence anthropometric traits, such as BMI,
\end{abstract}


body weight, and body adiposity index. Our findings also showed a dose-effect relationship between PPARGC1A rs8192678 and fasting plasma glucose. Finally, 5 rare mutations were identified in FNDC5, and 1 of these is a novel missense mutation. Conclusion: This study shows that genetic variants in the activation of brown-like adipocyte pathway play an important role in the susceptibility to severe obesity.

\section{Introduction}

Obesity (body mass index $[\mathrm{BMI}] \geq 30$ ) is a worldwide epidemic, affecting populations from developed and developing countries. Recent data show that $>1.9$ billion adults are overweight and there are at least 650 million people with obesity worldwide [1]. Epidemiological studies have shown that individuals with a very high BMI, e.g., subjects with severe obesity (BMI $\geq 35$ ) and individuals with morbid obesity (BMI $\geq 40$ ), have a substantial increased risk of comorbidities and mortality. Currently, the worldwide prevalence of severe obesity is nearly $3.7 \%$ and that of morbid obesity is $1.1 \%$. This continues to increase at alarming rates, with global projections estimating that severe obesity prevalence will reach $7.5 \%$ by 2025 [2, 3]. In Brazil, 2.2 million men (3.8\%) and 6.7 million women (5.3\%) had severe obesity in 2014 [2]. This pathology has emerged as one of the most prominent health care system problem in the world, since subjects with severe and morbid obesity incur between 45-113\% higher medical costs compared to normal-weight individuals [4-6]. Extreme BMI has been associated with a loss of life expectancy of 6.5-13.7 years [3].

The elevated prevalence of obesity is mostly due to the lifestyle changes in the last decades. These changes have resulted in an increased consumption of hypercaloric foods and reduced physical activity [7]. Energy expenditure, especially during exercise, plays an important role in combatting the development of obesity and related features. Regular physical activity benefits several tissues and improves quality of life; however, the precise molecular aspects underlying this mechanism are still unknown [8-10]. Boström et al. [11] reported a key role of brown-like adipocytes ("beige cells") in mice. During exercise, the peroxisome proliferator-activated receptor $\gamma$ coactivator $1 \alpha$ gene $(P P A R G C 1 A)$ is overexpressed in skeletal muscles, stimulating an increase in fibronectin type III domain containing 5 (FNDC5) gene expression. FNDC5 encodes a muscle membrane protein which is cleaved to release a newly identified hormone into the bloodstream, termed irisin. This novel myokine appears to act on white adipose tissue in mice and humans, stimulating browning through an increase in uncoupling protein 1 (UCP1) gene expression. This protein promotes the uncoupling of protons $\left(\mathrm{H}^{+}\right)$used for ATP synthesis during the mitochondrial respiratory chain, dissipating energy in the form of heat [12]. As a result, irisin activates adipocyte thermogenic programs, leading to mitochondrial heat production and energy expenditure $[10,11]$.

Irisin is a soluble small polypeptide containing 111 amino acids $(22 \mathrm{kDa})$, resulting from FNDC5 proteolysis at amino acid positions 30 and 140 [11, 13]. Since irisin was discovered, much research has focused on exploring its role in pathological and physiological conditions. Altered levels of irisin in the circulation have been previously associated with human obesity, but this association is inconsistent throughout the literature [14-17]. Demirpence et al. [17] showed that subjects with morbid obesity have lower levels of irisin compared to normal-weight subjects. Irisin was also found to be reduced in patients with type 2 diabetes and metabolic syndrome [18, 19]. Animal studies have suggested that increased plasma levels of irisin are associated with a decrease in body weight and an increase in energy expenditure [11]. Zhang et al. [13] conducted in vivo 
experiments to examine the potential therapeutic effects of irisin. After 14 days of daily injections of human recombinant irisin, fat mice showed reduced body weight and fasting insulin, and improved glucose tolerance. This result was accompanied by an increased number of multilocular subcutaneous adipocytes and the expression of UCP1, which suggests brown-like adipocyte development. All these findings indicate that irisin plays a key role in adiposity changes.

In this context, genetic variations in PPARGC1A, UCP1, and FNDC5 may be associated with obesity or related obesity-traits. The chromosomal region (4p15.1) in which PPARGC1A is located, has been linked with BMI in Mexican Americans [20] and abdominal fat in the Québec Family Study [21]. Furthermore, PPARGC1A polymorphisms have been associated with the development of obesity in different populations [22, 23]. Franks et al. [23] reported that the PPARGC1A rs8192678 polymorphism is associated with the accumulation of subcutaneous adiposity. Albuquerque et al. [24] observed a near nominal association between rs8192678 and body weight among Portuguese children. Interestingly, Pihlajamäki et al. [25] observed two haplotype blocks in PPARGC1A, one of which was located in the coding region and 3'UTR region and included the rs2970847 (Thr394 =), rs8192678 (Gly482Ser), rs3755863 (Thr528=), and rs3736265 (Thr612Met) polymorphisms. They found that this haplotype block was associated with glucose tolerance, BMI, and insulin sensitivity in patients with type 2 diabetes.

Ramos et al. [26] investigated the association between the UCP1 rs6536991 and rs12502572 polymorphisms and morbid obesity in a cohort of Brazilian population. They reported that rs6536991 was associated with BMI. Interestingly, both polymorphisms are located in the intronic region and it is unclear whether they affect UCP1 expression. Nishimura et al. [27] found that rs 12502572 has an effect on nonshivering thermogenesis, which might contribute to body weight regulation. To date, only a few studies have investigated the association between FNDC5 polymorphisms and obesity. Al-Daghri et al. [28] showed that the FNDC5 rs3480 polymorphism is associated with a decreased risk of obesity and a lower BMI in the Saudi population. Tang et al. [29] found that the rs16835198 polymorphism is associated with high-density lipoprotein (HDL)-cholesterol levels in individuals with overweight/obesity. These studies have focused on previously described common variants. However, there is a lack of information about rare and/or novel mutations.

Therefore, the aim of this study was to evaluate whether genetic variants in PPARGC1A, $U C P 1$, and FNDC5 are associated with severe obesity and related obesity traits in a cohort of adults from Rio de Janeiro, Southeast of Brazil. We postulated that these polymorphisms may affect protein functions or gene expression efficiency and could contribute to several pathophysiological conditions in severe obesity.

\section{Subjects}

This case-control, cross-sectional study comprised 401 adult individuals $(70.8 \%$ female and 29.2\% male), aged 18-65 years (median 35.0 [range 26.0-44.0] years), from the Southeast of Brazil. The participants were divided into 2 groups according to their BMI. Exclusion criteria were pregnancy, lactation, and the use of medication to lose or gain weight. The group with severe obesity (BMI $\geq 35.0$ ) consisted of 210 individuals recruited from a nongovernmental organization (the "Grupo de Resgate à Autoestima e Cidadania do Obeso" [GRACO]). These patients were candidates for bariatric surgery. The group of normal-weight subjects (BMI $\geq 18.5$ and $\leq 24.9$ ) included 191 participants who were volunteers from public hospitals in Rio de Janeiro. 


\section{Materials and Methods}

\section{Anthropometric Parameters}

The measurement of height, body weight, and waist and hip circumference was made according to classical methods. Briefly, waist circumference was evaluated at the midpoint between the iliac crest and the last costal arch, and hip circumference was measured at the level of the greater trochanters. BMI, body adiposity index (BAI), waist-to-weight ratio (WWR), and waist-to-hip ratio (WHR) were then calculated for each participant. BAI is a parameter used to estimate the percentage of body fat for adult men and women and is calculated using the formula: hip circumference/(height ${ }^{1.5}$ ) - 18 [30].

\section{Biochemistry}

Glucose, total cholesterol (TC), HDL-cholesterol (HDL-c), and triglycerides (TG) were measured by oxidase-peroxidase method (BioSystems), after an overnight fast. C-reactive protein (CRP) was evaluated using the latex agglutination method and glycated hemoglobin was measured by turbidimetric inhibition immunoassay (TINIA). Low-density lipoprotein cholesterol (LDL-c) was calculated by Friedewald formula (LDL-c $=\mathrm{TC}-\mathrm{HDL}-\mathrm{c}-\mathrm{TG} / 5$ ). Biochemical measurements influenced by medications taken by participants were not used in this statistical analysis.

\section{Genotyping}

Peripheral blood was collected from each participant and genomic DNA was extracted using a commercial DNA extraction kit (QIAamp Blood Kit, Qiagen, Valencia, CA, USA). Genotypes for the PPARGC1A (rs8192678, rs3736265, rs2970847, and rs3755863) and UCP1 (rs6536991 and rs12502572) polymorphisms were analyzed by real-time PCR using TaqMan ${ }^{\circledR}$ assays (ThermoFisher, Foster City, CA, USA). Amplification was performed in a StepOne ${ }^{\circledR}$ Plus real-time PCR system (ThermoFisher) using the manufacturer's protocol. Negative (all components excluding DNA) and positive controls were included for the genotyping quality conformation. We duplicated the test in $10 \%$ of the sample and there was $100 \%$ consistency.

For the FNDC5 gene, screening of exons 3-5 as well as their intron-exon boundaries was performed using Sanger automatic sequencing. These regions encode important structural regions of FNDC5, including the transmembrane domain, the targeting signal, and the site cleaved by irisin. These exons were amplified by PCR using 3 sets of custom-designed primers: exon 3 forward primer, 5'-TGGTCACCGAGTGTTGACAG-3' and reverse 5'-GGATAAGGGGGAGGAGACAG-3'; exon 4 forward primer, 5'-TGTGTGGGCACCTGTAGAAA-3' and reverse 5'-TCCAAACCCCTTACCCTTTT-3'; and exon 5 forward primer, 5'-AGAGACCATTGGCAAGCACT- $3^{\prime}$ and reverse $5^{\prime}$-GTCCAGGGATTACCAGAGCA-3'. Reactions were performed in a total volume of $25 \mu \mathrm{L}$, which included 20-50 ng of genomic DNA, 1.5 units of AmpliTaq Gold (ThermoFisher), $1 \times$ AmpliTaq buffer, $0.2 \mathrm{mmol} / \mathrm{L}$ of each dDNTP, $0.3 \mathrm{pM}$ of each primer and $2 \mathrm{mmol} / \mathrm{L} \mathrm{MgCl}_{2}$. Amplification of exon 3 was carried out under the following conditions: $95^{\circ} \mathrm{C}$ for $10 \mathrm{~min}$, followed by 35 cycles of $94^{\circ} \mathrm{C}$ for $1 \mathrm{~min}, 68^{\circ} \mathrm{C}$ for $1 \mathrm{~min}$ and $72^{\circ} \mathrm{C}$ for $1 \mathrm{~min}$; then an elongation step at $72^{\circ} \mathrm{C}$ for $10 \mathrm{~min}$. For exons 4 and 5 , the only difference was in the temperature used for annealing, i.e., $60^{\circ} \mathrm{C}$. PCR products were visualized on $1.5 \%$ agarose gels and purified with an Exosap kit (ThermoFisher) according to the manufacturer's protocol. Finally, sequencing was performed using reactions with $10 \mu \mathrm{L}$, containing 10-40 ng of PCR products, sequencing buffer $1 \times, 1.0 \mu \mathrm{L}$ big dye terminator kit v3.1 and $0.32 \mathrm{pm}$ of primer. The sequencing of products was carried out with the following conditions: 40 cycles of $94^{\circ} \mathrm{C}$ for $10 \mathrm{~s}, 50^{\circ} \mathrm{C}$ for $5 \mathrm{~s}$, and $60^{\circ} \mathrm{C}$ for $4 \mathrm{~min}$. Sequencing analysis was performed on an ABI Prism $3730 x l$ analyzer (ThermoFisher). The amplicons were sequenced bidirectionally and aligned with the sequence provided by the National Centre for Biotechnology Information (accession No. NM_001171940) using the software SeqScape v2.1 (ThermoFisher). 


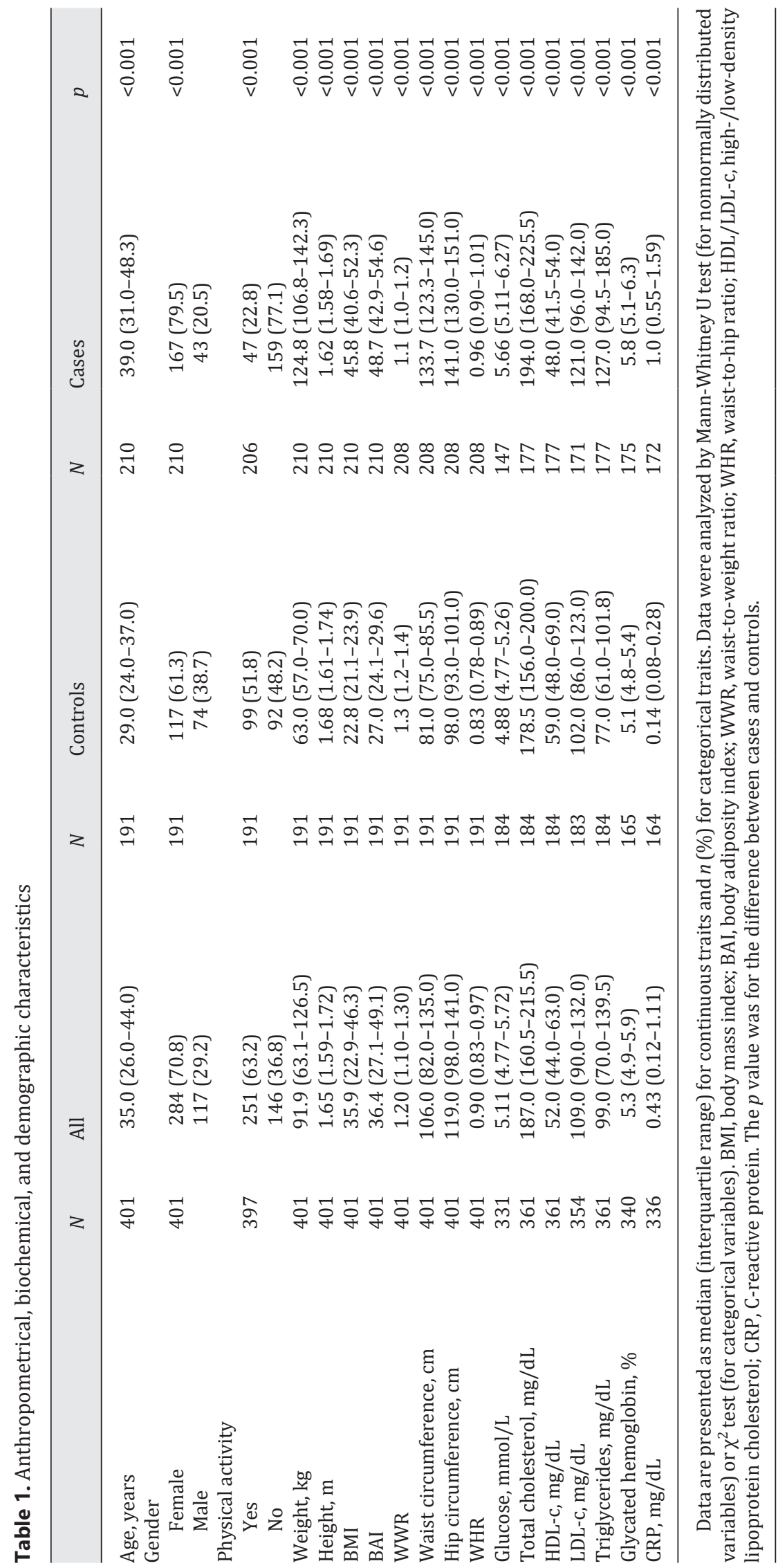




\section{Bioinformatic Tools}

FNDC5 sequences (genomic, transcript, and protein) were obtained from the National Centre for Biotechnology Information (https://www.ncbi.nlm.nih.gov/) and the Ensembl database (http://www.ensembl.org/). Additionally, the UniProt database was used to acquire information on the protein structure (http://www.uniprot.org/). PolyPhen and SIFT were selected to examine the potential pathogenicity of missense variants identified in this study (http://www.ensembl.org/info/docs/tools/vep/). Synonymous mutations were evaluated using FAS-ESS software which predicts the potential exonic splicing elements (http://genes.mit.edu/fas-ess/). Finally, intronic mutations of FNDC5 were explored using a splice-site prediction interface in NNSplice (http://www.fruitfly.org/seq_tools/splice. html).

\section{Statistical Analysis}

Normality of continuous parameters was tested with the Kolmogorov-Smirnov and Shapiro-Wilk tests. All continuous parameters were shown as nonnormal distribution. Differences in clinical, biochemical, and anthropometric characteristics between the case and control groups were calculated with the Mann-Whitney $\mathrm{U}$ and $\chi^{2}$ tests.

Genotype and allele distributions were calculated by gene counting. The Hardy-Weinberg equilibrium (HWE) was verified for each polymorphism using the $\chi^{2}$ test. Logistic regression analyses were performed to evaluate the association between PPARGC1A and UCP1 polymorphisms with severe obesity susceptibility, adjusted for gender and age. These association analyses were carried out in the additive, dominant, and recessive models. Moreover, the FNDC5 variants found in the case and control groups had association analyses performed with the $\chi^{2}$ test.

Continuous variables with nonnormal distribution were log-transformed before linear regression. The traits of interest (biochemical and anthropometric parameters) were chosen as dependent variables and polymorphism genotypes as independent variables (additive model). Gender and age were used as possible confounding variables for BMI, body weight, BAI, and WWR. Gender, age, and BMI were used as possible confounding variables for all other biochemical and anthropometric measurements. The analyses were performed using the SPSS statistical package (IBM, Chicago, IL, USA). $p<0.05$ was considered statistically significant.

In order to evaluate the sample size, our group used an iterative process to compute the minimum number of participants required to test the difference between 2 groups of qualitative variables (cases vs. controls). In this study, different polymorphisms were analyzed by selection of a conservative and a convenience sample ( $80 \%$ of statistical power) [31].

\section{Results}

Clinical characteristics of the 401 participants are shown in Table 1 . The cohort consisted of 210 subjects with severe obesity (i.e., cases; median BMI 45.6 [range 40.5-52.2]) and 191 individuals of normal-weight (i.e., controls; BMI 22.8 [21.1-23.9]). Generally, as expected, cases had higher anthropometric and biochemical data values than controls; exceptions were height, HDL-c, and WWR, for which the control group had higher values.

All samples were genotyped for polymorphisms in PPARGC1A (rs8192678, rs3736265, rs2970847, and rs3755863) and UCP1 (rs6536991 and rs12502572). The sample details about the genotype and allele frequencies are shown in Table 2. Genotypes of the polymorphisms were in HWE $(p>0.05)$ for both case and control groups. Association analyses showed that genotype and allele frequencies of PPARGC1A rs2970847 and UCP1 rs12502572 differed 
Table 2. Genotype and allele frequencies of PPARGC1A and UCP1 polymorphisms, and the risk for severe obesity

\begin{tabular}{|c|c|c|c|c|c|c|c|}
\hline \multirow[t]{2}{*}{ Gene } & \multirow[t]{2}{*}{ Polymorphism } & \multirow{2}{*}{$\begin{array}{l}\text { Controls } \\
(n=191)\end{array}$} & \multirow{2}{*}{$\begin{array}{l}\text { Cases } \\
(n=210)\end{array}$} & \multicolumn{2}{|c|}{ Odds ratio ( $95 \%$ confidence interval) } & \multirow[t]{2}{*}{$p$} & \multirow[t]{2}{*}{$p 1$} \\
\hline & & & & unadjusted & adjusted & & \\
\hline \multirow[t]{45}{*}{ PPARGC1A } & rs8192678 & & & & & & \\
\hline & Genotype & & & & & & \\
\hline & GG & $100(52.4)$ & $114(54.3)$ & 1.00 (ref.) & 1.00 (ref.) & - & - \\
\hline & GA & $78(40.8)$ & $82(39.0)$ & $0.92(0.61-1.39)$ & $0.98(0.63-1.53)$ & 0.699 & 0.927 \\
\hline & $\mathrm{AA}$ & $13(6.8)$ & $14(6.7)$ & $0.94(0.42-2.10)$ & $0.81(0.34-1.96)$ & 0.889 & 0.648 \\
\hline & Dominant model & & & & & & \\
\hline & GG & $100(52.4)$ & $114(54.3)$ & 1.00 (ref.) & 1.00 (ref.) & - & - \\
\hline & $\mathrm{GA}+\mathrm{AA}$ & $91(47.6)$ & $96(45.7)$ & $0.92(0.62-1.37)$ & $0.95(0.62-1.46)$ & 0.699 & 0.830 \\
\hline & Recessive model & & & & & & \\
\hline & $\mathrm{GG}+\mathrm{GA}$ & $178(93.2)$ & $196(93.3)$ & 1.00 (ref.) & 1.00 (ref.) & - & - \\
\hline & $\mathrm{AA}$ & $13(6.8)$ & $14(6.7)$ & $0.98(0.45-2.13)$ & $0.82(0.35-1.94)$ & 0.956 & 0.655 \\
\hline & Allele & & & & & & \\
\hline & $\mathrm{G}$ & $278(72.8)$ & $310(73.8)$ & 1.00 (ref.) & 1.00 (ref.) & - & - \\
\hline & A & $104(27.2)$ & $110(26.2)$ & $0.95(0.69-1.30)$ & $0.94(0.66-1.33)$ & 0.738 & 0.724 \\
\hline & rs3736265 & & & & & & \\
\hline & Genotype & & & & & & \\
\hline & GG & $166(87.0)$ & $188(89.5)$ & 1.00 (ref.) & 1.00 (ref.) & - & - \\
\hline & $\mathrm{GA}+\mathrm{AA}$ & $25(13.0)$ & $22(10.5)$ & $0.78(0.42-1.43)$ & $0.82(0.43-1.57)$ & 0.417 & 0.553 \\
\hline & Allele & & & & & & \\
\hline & $\mathrm{G}$ & 355 (92.9) & $398(94.8)$ & 1.00 (ref.) & 1.00 (ref.) & - & - \\
\hline & A & $27(7.1)$ & $22(5.2)$ & $0.73(0.41-1.30)$ & $0.77(0.42-1.42)$ & 0.287 & 0.408 \\
\hline & rs2970847 & & & & & & \\
\hline & Genotype & & & & & & \\
\hline & $\mathrm{CC}$ & $142(74.3)$ & $180(85.7)$ & 1.00 (ref.) & 1.00 (ref.) & - & - \\
\hline & $\mathrm{CT}$ & $46(24.1)$ & $29(13.8)$ & $0.50(0.30-0.83)$ & $0.48(0.27-0.84)$ & 0.008 & 0.010 \\
\hline & $\mathrm{TT}$ & $3(1.6)$ & $1(0.5)$ & $0.26(0.03-2.56)$ & $0.27(0.03-2.83)$ & 0.250 & 0.276 \\
\hline & Dominant model & & & & & & \\
\hline & $\mathrm{CC}$ & $142(74.3)$ & $180(85.7)$ & 1.00 (ref.) & 1.00 (ref.) & - & - \\
\hline & $\mathrm{CT}+\mathrm{TT}$ & 49 (25.7) & $30(14.3)$ & $0.48(0.29-0.80)$ & $0.46(0.27-0.80)$ & 0.005 & 0.006 \\
\hline & $\begin{array}{l}\text { Recessive model } \\
\mathrm{CC}+\mathrm{CT}\end{array}$ & $188(98.4)$ & $209(99.5)$ & 1.00 (ref) & 1.00 (ref) & _- & _- \\
\hline & TT & $3(1.6)$ & $1(0.5)$ & $0.31(0.03-3.23)$ & $0.30(0.03-2.91)$ & 0.328 & 0.299 \\
\hline & Allele & & & & & & \\
\hline & $\mathrm{C}$ & $330(86.4)$ & $389(92.6)$ & 1.00 (ref.) & 1.00 (ref.) & - & - \\
\hline & $\mathrm{T}$ & $52(13.6)$ & $31(7.4)$ & $0.50(0.31-0.80)$ & $0.48(0.29-0.81)$ & 0.004 & 0.006 \\
\hline & rs3755863 & & & & & & \\
\hline & Genotype & & & & & & \\
\hline & GG & $78(41.0)$ & $94(44.8)$ & 1.00 (ref.) & 1.00 (ref.) & - & - \\
\hline & GA & $90(47.0)$ & $84(40.0)$ & $0.78(0.51-1.18)$ & $0.84(0.53-1.32)$ & 0.236 & 0.443 \\
\hline & $\mathrm{AA}$ & $23(12.0)$ & $32(15.2)$ & $1.15(0.63-2.13)$ & $1.16(0.59-2.27)$ & 0.647 & 0.666 \\
\hline & Dominant model & & & & & & \\
\hline & GG & 78 (41.0) & $94(44.8)$ & 1.00 (ref.) & 1.00 (ref.) & - & - \\
\hline & $\mathrm{GA}+\mathrm{AA}$ & $113(59.2)$ & $116(55.2)$ & $0.85(0.57-1.27)$ & $0.90(0.59-1.39)$ & 0.428 & 0.639 \\
\hline & Recessive model & & & & & & \\
\hline & $\mathrm{GG}+\mathrm{GA}$ & $168(88.0)$ & $178(84.8)$ & 1.00 (ref.) & 1.00 (ref.) & - & - \\
\hline & $\mathrm{AA}$ & $23(12.0)$ & $32(15.2)$ & $1.31(0.74-2.33)$ & $1.27(0.68-2.38)$ & 0.354 & 0.456 \\
\hline
\end{tabular}


Table 2 (continued)

\begin{tabular}{|c|c|c|c|c|c|c|c|}
\hline \multirow[t]{2}{*}{ Gene } & \multirow[t]{2}{*}{ Polymorphism } & \multirow{2}{*}{$\begin{array}{l}\text { Controls } \\
(n=191)\end{array}$} & \multirow{2}{*}{$\begin{array}{l}\text { Cases } \\
(n=210)\end{array}$} & \multicolumn{2}{|c|}{ Odds ratio ( $95 \%$ confidence interval) } & \multirow[t]{2}{*}{$p$} & \multirow[t]{2}{*}{$p 1$} \\
\hline & & & & unadjusted & adjusted & & \\
\hline & Allele & & & & & & \\
\hline & G & 246 & 272 & 1.00 (ref.) & 1.00 (ref.) & - & - \\
\hline & A & 136 & 148 & $0.98(0.74-1.30)$ & $1.00(0.74-1.37)$ & 0.916 & 0.974 \\
\hline \multirow[t]{28}{*}{ UCP1 } & rs12502572 & & & & & & \\
\hline & Genotype & & & & & & \\
\hline & GG & $67(35.1)$ & $54(25.7)$ & 1.00 (ref.) & 1.00 (ref.) & - & - \\
\hline & GA & $89(46.6)$ & $106(50.5)$ & $1.48(0.94-2.33)$ & $1.44(0.87-2.36)$ & 0.093 & 0.152 \\
\hline & $\mathrm{AA}$ & $35(18.3)$ & $50(23.8)$ & $1.77(1.01-3.10)$ & $1.82(0.99-3.35)$ & 0.046 & 0.055 \\
\hline & Dominant model & & & & & & \\
\hline & GG & $67(35.1)$ & $54(25.7)$ & 1.00 (ref.) & 1.00 (ref.) & - & - \\
\hline & $\mathrm{GA}+\mathrm{AA}$ & $124(64.9)$ & $156(74.3)$ & $1.56(1.02-2.40)$ & $1.54(0.97-2.46)$ & 0.042 & 0.070 \\
\hline & Recessive model & & & & & & \\
\hline & $\mathrm{GG}+\mathrm{GA}$ & $156(81.7)$ & $160(76.2)$ & 1.00 (ref.) & 1.00 (ref.) & - & - \\
\hline & $\mathrm{AA}$ & 35 (18.3) & $50(23.8)$ & $1.39(0.86-2.26)$ & $1.45(0.86-2.46)$ & 0.181 & 0.167 \\
\hline & Allele & & & & & & \\
\hline & $\mathrm{G}$ & $223(58.4)$ & $214(51.0)$ & 1.00 (ref.) & 1.00 (ref.) & - & - \\
\hline & $\mathrm{A}$ & $159(41.6)$ & $206(49.0)$ & $1.34(1.02-1.78)$ & $1.36(1.00-1.84)$ & 0.037 & 0.049 \\
\hline & rs6536991 & & & & & & \\
\hline & Genotype & & & & & & \\
\hline & $\mathrm{TT}$ & $80(41.9)$ & $72(34.3)$ & 1.00 (ref.) & 1.00 (ref.) & - & - \\
\hline & $\mathrm{TC}$ & $84(44.0)$ & $104(49.5)$ & $1.38(0.90-2.11)$ & $1.22(0.77-1.95)$ & 0.145 & 0.397 \\
\hline & $\mathrm{CC}$ & $27(14.1)$ & $34(16.2)$ & $1.40(0.77-2.54)$ & $1.30(0.68-2.48)$ & 0.270 & 0.431 \\
\hline & Dominant model & & & & & & \\
\hline & TT & 80 (41.9) & $72(34.3)$ & 1.00 (ref.) & 1.00 (ref.) & - & - \\
\hline & $\mathrm{TC}+\mathrm{CC}$ & $111(58.1)$ & $138(65.7)$ & $1.38(0.92-2.07)$ & $1.24(0.80-1.92)$ & 0.118 & 0.336 \\
\hline & Recessive model & & & & & & \\
\hline & $\mathrm{TT}+\mathrm{TC}$ & $164(85.9)$ & $176(83.8)$ & 1.00 (ref.) & 1.00 (ref.) & - & - \\
\hline & $\mathrm{CC}$ & 27 (14.1) & 34 (16.2) & $1.17(0.68-2.03)$ & $1.16(0.64-2.10)$ & 0.568 & 0.624 \\
\hline & Allele & & & & & & \\
\hline & $\mathrm{T}$ & $244(63.9)$ & $248(59.0)$ & 1.00 (ref.) & 1.00 (ref.) & - & - \\
\hline & $\mathrm{C}$ & $138(36.1)$ & $172(41.0)$ & $1.16(0.85-1.58)$ & $1.22(0.92-1.63)$ & 0.354 & 0.164 \\
\hline
\end{tabular}

Odds ratios and $p$ value were calculated using logistic regression. ${ }^{1}$ Adjusted for gender and age.

${ }^{a}$ No homozygous AA in case subjects was identified.

significantly between the groups. The frequency of rs2970847 wild-type genotype (CC) was significantly higher in the case group than in the control group. In order to explore this association, dominant and recessive models were performed. PPARGC1A rs2970847 was significantly associated with severe obesity in a dominant model. Allelic analysis showed that the rs2979847 (C) allele was more frequent in the subjects with severe obesity than in the individuals of normal weight ( 7.4 vs. $13.7 ; p=0.004)$. Furthermore, individuals carrying the rs2979847 (C) allele had a 2.01-fold increased risk of severe obesity compared to subjects without this allele.

The frequency of UCP1 rs12502572 (AA) genotype was higher in the cases than in the controls. Moreover, our result demonstrated that this polymorphism was associated with obesity in a dominant model. After adjustment for age and gender, these associations were 


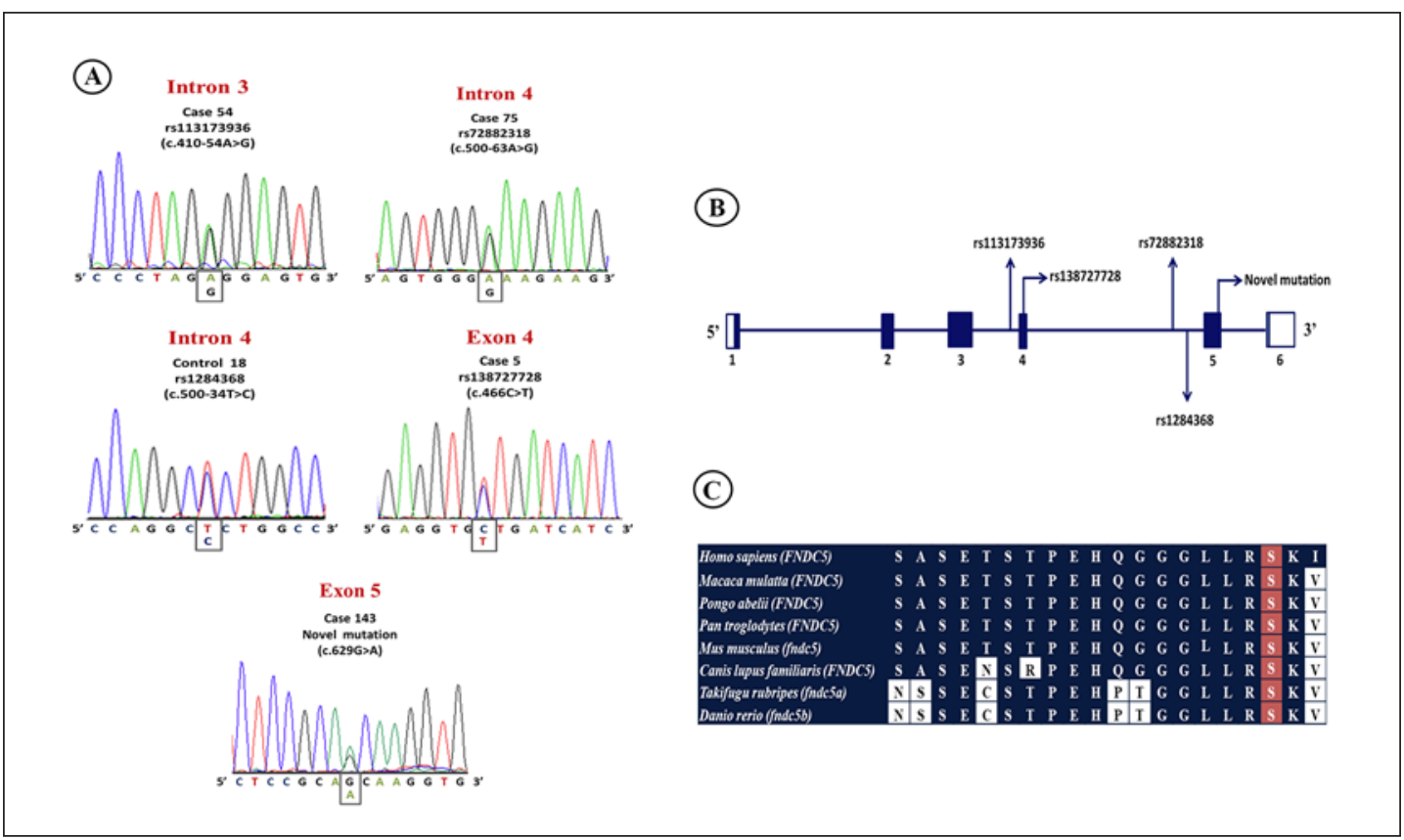

Fig. 1. A Electropherograms of the FNDC5 gene. B FNDC5 structure and position of the 5 mutations identified by Sanger sequencing. C A portion of the amino acid sequence of FNDC5 in diverse species. The position of the new mutation is showed in the red squares, suggesting it is highly conserved in different organisms. The nonconserved position in specific species is shown in the white squares.

no longer observed. However, allelic analysis showed that the minor allele (A) frequency was higher in subjects with severe obesity than in controls ( 49.0 vs. $41.6 ; p=0.037$ ). Therefore, for individuals carrying the UCP1 rs12502572 (A) allele, the risk of developing severe obesity increased 1.34-fold. No association was found between obesity and PPARGC1A (rs8192678, rs3736265, and rs3755863) or UCP1 (rs6536991).

The influence of $P P A R G C 1 A$ and $U C P 1$ polymorphisms on anthropometric and biochemical measurements in our sample was analyzed (online suppl. data, Table S1; for all online suppl. material, see www.karger.com/doi/10.1159/000505666). PPARGC1A rs2970847 and UCP1 rs12502572 were associated with body weight, BMI, and BAI in an additive model. PPARGC1A rs2970847 was also associated with WWR. In addition, PPARGC1A rs8192678 had an influence on plasma glucose measurements. The additive effect on variables was found for the UCP1 rs12502572 and PPARGC1A rs8192678 polymorphisms. Our results showed that the median value increased according to the number of risk alleles, so that subjects carrying 2 risk alleles had higher median values. Our results indicated no significant effects of PPARGC1A rs 3755863 , PPARGC1A rs3736265, and UCP1 rs6536991 polymorphisms on anthropometric and biochemical levels.

The FNDC5 gene was screened in order to investigate the prevalence of mutations in the severe obesity and normal-weight groups. We identified 5 rare mutations in the heterozygous state (Fig. 1A). The prevalence of these variations in the case and control groups is shown in Table 3. Three single-nucleotide substitutions were located at the intron-exon boundaries (rs113173936, rs72882318, and rs1284368). These variants have been described in the literature and were found in both cases and controls. Intronic mutations may not affect the protein sequence but have a potential pathogenic effect if they disrupt splicing mechanisms. These variants were thus examined using a splice-site prediction interface. It demonstrated 
that these mutations were not located in acceptor or donor sites. The association between the variants and severe obesity was evaluated in our sample, but no significant result was found.

Only 2 subjects with severe obesity showed variants in the coding region, one with a synonymous mutation (rs138727728) and the other with a novel missense mutation (Fig. 1B). The synonymous mutation is localized at exon 4 of the FNDC5 gene, where cytosine is substituted for thymine at the base position 466 (Leu81 =). Despite not affecting the amino acid, this type of mutation may disturb important regulatory elements. We therefore examined the potential exonic splicing enhancers or silencers in the FNDC5 gene. The results showed that the mutation was not localized in these regions.

The novel mutation is localized at exon 5 of FNDC5, namely c.629G $>$ A (Ser210Asn). The potential pathogenicity of this mutation was evaluated using in silico software. The Polyphen software suggested that the mutation was probably damaging (score: 0.916). The SIFT software result indicated that variation was deleterious with low confidence (score: 0.03 ). Furthermore, this residue is highly conserved between different species (from humans to zebrafish) (Fig. 1C).

\section{Discussion}

In this study, we explored the genetic variations in the PPARGC1A, UCP1, and FNDC5 genes as potential risk factors for the development of severe obesity in a Brazilian population. Additionally, we evaluated the association between these polymorphisms and obesity-related traits. To this end, we genotyped individuals with severe obesity $(n=210)$ and normal-weight controls $(n=191)$ for common polymorphisms in the PPARGC1A and UCP1 genes. Our results indicated that PPARGC1A rs2970847 and UCP1 rs12502572 are associated with a susceptibility to severe obesity and obesity-related parameters. Moreover, PPARGC1A rs8192678 is associated with an increase in glucose plasma levels. We also screened exons 3-5 (including their intron-exon boundaries) of the FNDC5 gene, and identified 5 rare mutations, 1 of them a novel missense mutation (Ser210Asn).

The PPARGC1A gene encodes a transcriptional coactivator protein, which plays a key role in energy metabolism via mitochondrial biogenesis, fatty acid oxidation, hepatic gluconeogenesis, glucose uptake, and lipid metabolism [32-35]. In this study, we examined 4 common polymorphisms, and found that the PPARGC1A rs2970847 polymorphism is associated with severe obesity susceptibility. Our findings showed that individuals carrying the PPARGC1A rs2970847 (CC) genotype have an increased risk of becoming severely obese. Moreover, subjects with this genotype had higher median body weight, BMI, BAI, and WWR values.

PPARGC1A rs2970847 is a synonymous mutation (Thr394Thr), and it is unclear how this polymorphism could affect body metabolism to alter the risk for obesity in our sample. Interestingly, there is evidence that synonymous mutations are important for different aspects of human disease and therefore therapeutic approaches. These mutations may disturb basic cellular processes such as gene regulation, mRNA secondary structure, stability or splicing, and protein synthesis, folding, or functionality [36]. They could also be in complete or nearcomplete linkage disequilibrium with a pathogenic variant [37]. In the literature, there are only a few studies on PPARGC1A rs2970847 and obesity. Vimaleswaran et al. [37] reported that this polymorphism influences adiposity in Asian Indian subjects. They observed that visceral and subcutaneous fat are lower in subjects with the PPARGC1A rs2970847 (CC) genotype. In contrast, in our sample, individuals carrying the PPARGC1A rs2970847 (CC) genotype had higher adiposity values. The discrepancy may be explained by the differences in genetic backgrounds and sample criteria. 
da Fonseca et al.: Genetic Variants in the Energy Expenditure Metabolism

We also showed an association between PPARGC1A rs8192678 and fasting plasma glucose. PPARGC1A rs8192678 is a missense mutation which changes glycine to serine at codon 482 (Gly482Ser). Individuals carrying the risk allele (482Ser) have reduced levels of PPARGC1A mRNA compared to homozygous subjects [38]. Povel et al. [39] reported that the presence of the risk allele was associated with lower glucose levels in normal-weight individuals (BMI $\leq 25.0$ ). However, their results also suggested an association with the opposite trend in subjects with a higher BMI (BMI $\geq 28.0$ ), similar to our findings. Since the PGC1- $\alpha$ protein, encoded by PPARGC1A, acts on energy expenditure and fuel uptake, this polymorphism may affect energy balance. In this context, previous functional studies showed that PGC1- $\alpha$ regulates glucose uptake and oxidation via expression of the glucose transporter type 4 (GLUT4) gene in muscle [38, 40]. Therefore, we hypothesized that the higher glucose levels in our patients carrying the Ser482 allele could be explained by the reduced abundance of PGC1- $\alpha$ and GLUT4, which consequently resulted in decreased glucose uptake and metabolization. However, functional studies are necessary to confirm this hypothesis.

UCP1 is located in the inner mitochondrial membrane of brown and brown-like adipocytes, where it has a key role in thermogenesis and energy expenditure [11, 12, 41]. In this context, UCP1 is a strong candidate gene for obesity susceptibility, and several genetic studies have evaluated this association [26, 42, 43]. Our findings demonstrate that the UCP1 rs12502572 (A) allele is a risk factor for severe obesity. We observed an additive effect of this allele in the anthropometric parameters (body weight, BMI, and BAI).

To date, studies on the UCP1 rs12502572 polymorphism are scarce in the literature. Ramos et al. [26] selected 239 adult patients from Brazil (126 subjects with morbid obesity and 113 individuals with normal obesity) but found no association between the UCP1 rs12502572 polymorphism and obesity or related traits. This polymorphism is located at intron 2 and it is unknown whether it affects the gene expression of UCP1. Nishimura et al. [27] found that it has an effect on nonshivering thermogenesis caused by the generation of heat via the inhibition of mitochondrial ATP synthesis in brown tissues. Since thermoregulatory mechanisms contribute to the regulation of body weight and our results showed an association between UCP1 rs12502572 (A) allele and increased body adiposity, we suggest that UCP1 rs12502572 might affect gene expression and, consequently, energy expenditure. Further functional studies are required to examine how this polymorphism could affect UCP1 expression.

Finally, the main region of the FNDC5 gene, which encodes the transmembrane domain, targeting signal, and irisin-cleaved site, was sequenced in the case and control groups. Five rare mutations were identified; 3 were located at the intron-exon boundary and 2 in the coding region. Our results showed that intronic mutations as well as the synonymous mutation were not located in potential splicing elements, suggesting that they do not disturb this cellular mechanism. A novel missense mutation was found at exon 5 , which led to a serine-toasparagine substitution at codon 210 . The substituted amino acid is highly conserved in different species from human to zebrafish, and both Polyphen and SIFT software have predicted that it can have pathogenic effects.

FNDC5 is a type 1 transmembrane protein. This novel mutation is positioned in the cytoplasmic region, which has an important role in the microbody-targeting signal (UniProtKB database, ID: Q8NAU1). The C-terminal region of FNDC5 contains an important sequence to transport proteins into their correct microbody compartment. Moreover, some studies have reported that such regions are evolutionary conserved, demonstrating their important role in protein transportation $[44,45]$. Interestingly, the new mutation located in this region was found only in a female patient with extreme obesity (BMI 56.1), who had developed severe obesity during pregnancy. Since FNDC5 is cleaved and released as irisin, a hormone that has a key role in the activation of the browning pathway [11], we suggest that this novel mutation 
may impact on protein transport which could decrease energy expenditure and contribute to a susceptibility to severe obesity.

This study had some limitations that should be considered when interpreting the results. First, it had a cross-sectional study design and we were unable to exclude the fluctuations of biochemical and anthropometric traits. Second, the interaction between genes and lifestyle were not investigated and could be a confounding factor. Finally, a lack of data on the mRNA or protein expression of the studied genes meant we could not confirm that the genetic variants are in fact associated with modifications in expression.

\section{Conclusion}

Obesity is a complex disease resulting from an interaction of environmental and genetic factors. A number of previous studies have highlighted the important role of polymorphisms in some energy balance genes and their association with susceptibility to developing obesity, which might be helpful for establishing a personalized therapy and/or prevention. We investigated genetic variants in PPARGC1A, UCP1, and FNDC5, genes that are related to the activation of the brown-like adipocyte pathway. We identified that the PPARGC1A rs2970847 and UCP1 rs12502572 polymorphisms modulate the risk of developing severe obesity and obesity-related traits in a Brazilian population. Our findings also indicated an association of PPARGC1A rs8192678 and glucose plasma levels. The sequencing of the FNDC5 gene identified 5 rare variants, 1 a novel missense mutation located in the microbody-targeting signal. This mutation needs further functional analysis to clarify its potential pathogenic effect on FNDC5 transport and metabolism.

\section{Acknowledgements}

We are grateful to the patients and healthy volunteers who kindly agreed to participate in this study. We thank Nereida Proença da Fonseca for her great technical assistance with the participants and Rosimere Lima for her excellent work with patients in GRACO.

\section{Statement of Ethics}

The study protocol was performed according to the Declaration of Helsinki (1964) and approved by the Ethics Committee of the Oswaldo Cruz Foundation. All participants provided written consent prior to enrollment.

\section{Disclosure Statement}

There were no conflicts of interest.

\section{Funding Sources}

This work was supported by the Oswaldo Cruz Foundation (Fiocruz, Brazil) and the Brazilian Ministry of Health.

\section{Availability of Data}

The study data are available on request. 
da Fonseca et al.: Genetic Variants in the Energy Expenditure Metabolism

\section{References}

1 World Health Organization. [Internet] Obesity and overweight [cited 2 April 2019]. Available from: https:// www.who.int/news-room/fact-sheets/detail/obesity-and-overweight

2 Di Cesare M, Bentham J, Stevens GA, Zhou B, Danaei G, Lu Y, et al.; NCD Risk Factor Collaboration (NCD-RisC). Trends in adult body-mass index in 200 countries from 1975 to 2014: a pooled analysis of 1698 populationbased measurement studies with 19.2 million participants. Lancet. 2016 Apr;387(10026):1377-96.

3 Kitahara CM, Flint AJ, Berrington de Gonzalez A, Bernstein L, Brotzman M, MacInnis RJ, et al. Association between class III obesity (BMI of 40-59 kg/m2) and mortality: a pooled analysis of 20 prospective studies. PLoS Med. 2014 Jul;11(7):e1001673.

4 Arterburn DE, Maciejewski ML, Tsevat J. Impact of morbid obesity on medical expenditures in adults. Int J Obes. 2005 Mar;29(3):334-9.

5 Keating CL, Peeters A, Swinburn BA, Magliano DJ, Moodie ML. Utility-based quality of life associated with overweight and obesity: the Australian diabetes, obesity, and lifestyle study. Obesity (Silver Spring). 2013 Mar; 21(3):652-5.

6 Withrow D, Alter DA. The economic burden of obesity worldwide: a systematic review of the direct costs of obesity. Obes Rev. 2011 Feb;12(2):131-41.

7 da Fonseca AC, Mastronardi C, Johar A, Arcos-Burgos M, Paz-Filho G. Genetics of non-syndromic childhood obesity and the use of high-throughput DNA sequencing technologies. J Diabetes Complications. 2017 Oct; 31(10):1549-61.

8 Hruby A, Hu FB. The Epidemiology of Obesity: A Big Picture. Pharmacoeconomics. 2015 Jul;33(7):673-89.

9 Huh JY, Panagiotou G, Mougios V, Brinkoetter M, Vamvini MT, Schneider BE, et al. FNDC5 and irisin in humans: I. Predictors of circulating concentrations in serum and plasma and II. mRNA expression and circulating concentrations in response to weight loss and exercise. Metabolism. 2012 Dec;61(12):1725-38.

10 Kelly DP. Medicine. Irisin, light my fire. Science. 2012 Apr;336(6077):42-3.

11 Boström P, Wu J, Jedrychowski MP, Korde A, Ye L, Lo JC, et al. A PGC1- $\alpha$-dependent myokine that drives brownfat-like development of white fat and thermogenesis. Nature. 2012 Jan;481(7382):463-8.

12 Azzu V, Brand MD. The on-off switches of the mitochondrial uncoupling proteins. Trends Biochem Sci. 2010 May;35(5):298-307.

13 Zhang Y, Li R, Meng Y, Li S, Donelan W, Zhao Y, et al. Irisin stimulates browning of white adipocytes through mitogen-activated protein kinase p38 MAP kinase and ERK MAP kinase signaling. Diabetes. 2014 Feb;63(2): 514-25.

14 Crujeiras AB, Pardo M, Arturo RR, Navas-Carretero S, Zulet MA, Martínez JA, et al. Longitudinal variation of circulating irisin after an energy restriction-induced weight loss and following weight regain in obese men and women. Am J Hum Biol. 2014 Mar-Apr;26(2):198-207.

15 Fagundo AB, Jiménez-Murcia S, Giner-Bartolomé C, Agüera Z, Sauchelli S, Pardo M, et al. Modulation of Irisin and Physical Activity on Executive Functions in Obesity and Morbid obesity. Sci Rep. 2016 Aug;6(1):30820.

16 Novelle MG, Contreras C, Romero-picó A, López M, Diéguez C. Irisin, two years later. Int J Endocrinol. 2013; 2013:746281.

17 Demirpence M, Yilmaz H, Colak A, Yalcin H, Toprak B, Turkon H, et al. The effect of sleeve gastrectomy on serum irisin levels in patients with morbid obesity. Endokrynol Pol. 2016;67(5):481-6.

18 Liu JJ, Wong MD, Toy WC, Tan CS, Liu S, Ng XW, et al. Lower circulating irisin is associated with type 2 diabetes mellitus. J Diabetes Complications. 2013 Jul-Aug;27(4):365-9.

19 Yan B, Shi X, Zhang H, Pan L, Ma Z, Liu S, et al. Association of serum irisin with metabolic syndrome in obese Chinese adults. PLoS One. 2014 Apr;9(4):e94235.

20 Arya R, Duggirala R, Jenkinson CP, Almasy L, Blangero J, O'Connell P, et al. Evidence of a novel quantitativetrait locus for obesity on chromosome 4p in Mexican Americans. Am J Hum Genet. 2004 Feb;74(2):272-82.

21 Pérusse L, Rice T, Chagnon YC, Després JP, Lemieux S, Roy S, et al. A genome-wide scan for abdominal fat assessed by computed tomography in the Québec Family Study. Diabetes. 2001 Mar;50(3):614-21.

22 Hong MS, Kim HK, Shin DH, Song DK, Ban JY, Kim BS, et al. Integrative study on PPARGC1A: hypothalamic expression of Ppargc1a in ob/ob mice and association between PPARGC1A and obesity in Korean population. Mol Cell Toxicol. 2008;4:318-22.

23 Franks PW, Christophi CA, Jablonski KA, Billings LK, Delahanty LM, Horton ES, et al.; Diabetes Prevention Program Research Group. Common variation at PPARGC1A/B and change in body composition and metabolic traits following preventive interventions: the Diabetes Prevention Program. Diabetologia. 2014 Mar;57(3): 485-90.

24 Albuquerque D, Nóbrega C, Rodríguez-López R, Manco L. Association study of common polymorphisms in MSRA, TFAP2B, MC4R, NRXN3, PPARGC1A, TMEM18, SEC16B, HOXB5 and OLFM4 genes with obesity-related traits among Portuguese children. J Hum Genet. 2014 Jun;59(6):307-13.

25 Pihlajamäki J, Kinnunen M, Ruotsalainen E, Salmenniemi U, Vauhkonen I, Kuulasmaa T, et al. Haplotypes of PPARGC1A are associated with glucose tolerance, body mass index and insulin sensitivity in offspring of patients with type 2 diabetes. Diabetologia. 2005 Jul;48(7):1331-4.

26 Ramos AV, Bastos-Rodrigues L, Resende BA, Friedman E, Campanha-Versiani L, Miranda DM, et al. The contribution of FTO and UCP-1 SNPs to extreme obesity, diabetes and cardiovascular risk in Brazilian individuals. BMC Med Genet. 2012 Nov;13(1):101. 
da Fonseca et al.: Genetic Variants in the Energy Expenditure Metabolism

27 Nishimura T, Katsumura T, Motoi M, Oota H, Watanuki S. Experimental evidence reveals the UCP1 genotype changes the oxygen consumption attributed to non-shivering thermogenesis in humans. Sci Rep. 2017 Jul; $7(1): 5570$.

28 Al-Daghri NM, Mohammed AK, Al-Attas OS, Amer OE, Clerici M, Alenad A, et al. SNPs in FNDC5 (irisin) are associated with obesity and modulation of glucose and lipid metabolism in Saudi subjects. Lipids Health Dis. 2016 Mar; 15(1):54.

29 Tang S, Zhang R, Jiang F, Wang J, Chen M, Peng D, et al. An interaction between a FNDC5 variant and obesity modulates glucose metabolism in a Chinese Han population. PLoS One. 2014 Nov;9(11):e109957.

30 Bergman RN, Stefanovski D, Buchanan TA, Sumner AE, Reynolds JC, Sebring NG, et al. A better index of body adiposity. Obesity (Silver Spring). 2011 May;19(5):1083-9.

31 Zar JH, editor. Biostatistical Analysis. 4th ed. Upper saddle River (NJ): Prentice Hall; 1998.

32 Puigserver P, Spiegelman BM. Peroxisome proliferator-activated receptor-gamma coactivator 1 alpha (PGC-1 alpha): transcriptional coactivator and metabolic regulator. Endocr Rev. 2003 Feb;24(1):78-90.

33 Kunej T, Globocnik Petrovic M, Dovc P, Peterlin B, Petrovic D. A Gly482Ser polymorphism of the peroxisome proliferator-activated receptor-gamma coactivator-1 (PGC-1) gene is associated with type 2 diabetes in Caucasians. Folia Biol (Praha). 2004;50(5):157-8.

34 Medina-Gomez G, Gray S, Vidal-Puig A. Adipogenesis and lipotoxicity: role of peroxisome proliferator-activated receptor gamma (PPARgamma) and PPARgammacoactivator-1 (PGC1). Public Health Nutr. 2007 Oct;10 10A:1132-7.

35 da Fonseca ACP, Ochioni AC, Martins RDS, Zembrzuski VM, Campos Jr M, Ramos VG, et al. Adiponectin, Retinoic Acid Receptor Responder 2, and Peroxisome Proliferator-Activated Receptor- $\gamma$ Coativator- 1 Genes and the Risk for Obesity. Dis Markers. 2017;2017:5289120.

36 Hunt RC, Simhadri VL, Iandoli M, Sauna ZE, Kimchi-Sarfaty C. Exposing synonymous mutations. Trends Genet. 2014 Jul;30(7):308-21.

37 Vimaleswaran KS, Radha V, Anjana M, Deepa R, Ghosh S, Majumder PP, et al. Effect of polymorphisms in the PPARGC1A gene on body fat in Asian Indians. Int J Obes. 2006 Jun;30(6):884-91.

38 Ling C, Poulsen P, Carlsson E, Ridderstråle M, Almgren P, Wojtaszewski J, et al. Multiple environmental and genetic factors influence skeletal muscle PGC-1 $\alpha$ and PGC-1beta gene expression in twins. J Clin Invest. 2004 Nov; 114(10):1518-26.

39 Povel CM, Feskens EJ, Imholz S, Blaak EE, Boer JM, Dollé ME. Glucose levels and genetic variants across transcriptional pathways: interaction effects with BMI. Int J Obes. 2010 May;34(5):840-5.

40 Michael LF, Wu Z, Cheatham RB, Puigserver P, Adelmant G, Lehman JJ, et al. Restoration of insulin-sensitive glucose transporter (GLUT4) gene expression in muscle cells by the transcriptional coactivator PGC-1. Proc Natl Acad Sci USA. 2001 Mar;98(7):3820-5.

41 Bouillaud F, Villarroya F, Hentz E, Raimbault S, Cassard AM, Ricquier D. Detection of brown adipose tissue uncoupling protein mRNA in adult patients by a human genomic probe. Clin Sci (Lond). 1988 Jul;75(1):21-7.

42 Brondani LA, de Souza BM, Assmann TS, Bouças AP, Bauer AC, Canani LH, et al. Association of the UCP polymorphisms with susceptibility to obesity: case-control study and meta-analysis. Mol Biol Rep. 2014 Aug; 41(8):5053-67.

43 Nicoletti CF, de Oliveira AP, Brochado MJ, de Oliveira BP, Pinhel MA, Marchini JS, et al. UCP1 -3826 A[\{GT\}]G polymorphism affects weight, fat mass, and risk of type 2 diabetes mellitus in grade III obese patients. Nutrition. 2016 Jan;32(1):83-7.

44 Keller GA, Krisans S, Gould SJ, Sommer JM, Wang CC, Schliebs W, et al. Evolutionary conservation of a microbody targeting signal that targets proteins to peroxisomes, glyoxysomes, and glycosomes. J Cell Biol. 1991 Sep; 114(5):893-904.

45 Neupert W, Lill R, editors. Membrane Biogenesis and Protein Targeting. Elsevier; 1992. 